\title{
Study of the expression of Tn antigen in different types of human breast cancer cells using VVA-B ${ }_{4}$ lectin
}

\author{
G. KONSKA ${ }^{1}$, M. GUERRY ${ }^{2}$, F. CALDEFIE-CHEZET ${ }^{2}$, M. DE LATOUR ${ }^{3}$ and J. GUILLOT ${ }^{2}$ \\ ${ }^{1}$ Department of Pharmaceutical Botany, Collegium Medicum, Jagiellonian University, ul. Medyczna 9, 30-688 Cracow, \\ Poland; ${ }^{2}$ LBCM, EA2416, Centre de Recherche en Nutrition Humaine d'Auvergne, Faculté de Pharmacie, \\ ${ }^{3}$ Centre Anti-cancéreux Jean-Perrin, Centre Hospitalier Universitaire, Clermont-Ferrand, France
}

Received May 17, 2005; Accepted July 8, 2005

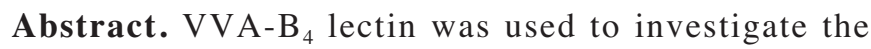
differences in Tn antigen expression in tissues of different types of human breast cancer (benign lesions, carcinoma in situ, invasive carcinoma) and in normal tissues neighboring lobular carcinoma. Locations in which Tn antigen was expressed were identified using the avidin-biotin-peroxidase labeling system. Tissues collected during cosmetic procedures and classified as normal were completely negative, except for one case. Benign proliferative changes including fibroadenoma, apocrine and cylindrical metaplasia showed a very weak positive reaction, although strongly positive cells were also observed. The reaction in non-invasive cases of atypical hyperplasia was diversified depending on site. Intralobular hyperplasia was characterized by a particularly high percentage of labeled cells. A majority (up to $80 \%$ ) of ductal and lobular carcinoma in situ showed very strong or moderate staining. In invasive cancers, there were conspicuous differences between stage of cancer development and tendency towards a decrease in intensely labeled cell count in the most advanced stages. In normal tissues in the direct neighborhood of carcinoma in situ, the cytoplasm of $40 \%$ of cells was strongly labeled. However, the findings for normal tissues in the close vicinity of invasive cancer were the most surprising, since there was either no or only very weak positive reaction. It can be concluded that glycosylation modifications during carcinogenesis, as demonstrated by the presence of Tn epitope, develop very early, before any destructive changes in proliferation/apoptosis or cell differentiation become discernible.
\end{abstract}

Correspondence to: Professor Jean Guillot, LBCM, EA2416, Faculté de Pharmacie, 28 Place Henri-Dunant, BP38, 63000 Clermont-Ferrand, France

E-mail: jean.guillot@u-clermont1.fr

Key words: Tn antigen, human breast tumors, lectin histochemistry, Vicia villosa lectin

\section{Introduction}

Oncofetal antigens, including $\mathrm{T}$ (Thomsen-Friedenreich) antigen and Tn antigen, occupy a prominent position among numerous known tumor markers (1). The structure of these antigens matches the basic core 1 structure of Golgi apparatus glycoproteins containing $O$-glycoside bond which comprise, among others, blood group antigens A, B, H and Lewis (2). $\mathrm{T}$ antigen structure can be identified in a molecule of glycophorine $\mathrm{A}^{\mathrm{M}}$ and glycophorine $\mathrm{A}^{\mathrm{N}}$ in the form of tetrasaccharide chains NeuAc- $\alpha 2-3$ Galß1-3[NeuAc $\alpha 2-6]$ GalNac $\alpha 1-l i n k e d$ through $O$-glycoside bonds with serine and threonine of the peptide chain. The sialylated form of Tn antigen (sTn) is formed due to a lack of B1-3 D-galactosyltransferase activity, while Tn antigen is created when the activities of the two enzymes - B1-3 D-galactosyltransferase and $\alpha 2-6$ sialyltransferase, are lacking $(3,4)$.

The appearance of a non-cryptic Tn antigen structure is observed in a short period of fetal life (5) in so-called Tn syndrome (6) and during the process of carcinogenesis (7). Its widespread presence has been noted on tumors of epithelial origin but it is absent on tumors of ectodermal and mesodermal origin (8). Studies using monoclonal antibodies and lectins have demonstrated the expression of sialylated and nonsialylated forms of Tn antigen in gastrointestinal tumors, particularly in colon cancer $(9,10)$, pancreatic $(11)$ and gastric (12) cancers, in ovarian (13) and uterine (14) cancers, and in numerous cases of breast cancer (14-18). Tn antigen mostly occurs in malignant breast lesions, invasive carcinomas and their metastases, but it has also been found in benign lesions. Except in very rare cases $(19,20)$, it is not detected in normal breast tissue.

Studies into the localization of Tn antigen have widely used lectins with appropriate sugar specificity, besides monoclonal antibodies. VVA-B , $_{4}$ one of three isolectins occurring in Vicia villosa (Fabaceae), belongs to the group of the lectins commonly used for this purpose. This lectin (108 kDa glycoprotein built of 4 identical subunits) specifically binds to the sugar sequence containing $N$-acetylgalactosamine at the chain end, and reacts only with human Tn antigen-exposed erythrocytes or erythrocytes with Cad specificity (21).

It has been demonstrated that neoplastic transformation of the cell leads to permanent or reversible blockade of $B 1-3$ 
Table I. Tn antigen expression in normal tissue and in different types of breast cancer.

\begin{tabular}{|c|c|c|c|c|}
\hline & Cancer type & No. of cases & Staining intensity ${ }^{\mathrm{a}}$ & Stained cells $(\%)$ \\
\hline \multirow[t]{2}{*}{ Normal tissues } & & 3 & $(0): 2$ cases & 0 \\
\hline & & & $(+): 1$ case & $>15$ \\
\hline \multirow[t]{4}{*}{ Benign changes } & Cylindrical metaplasia & 3 & $(0) \rightarrow(+)$ & $<20$ \\
\hline & Apocrine metaplasia & 7 & $(+) \rightarrow(+++)$ & 90 \\
\hline & Fibroadenoma & 2 & $(+)$ & 5 \\
\hline & & & $(++)$ & 40 \\
\hline \multirow[t]{3}{*}{ Atypical hyperplasia } & Intraductal hyperplasia & 4 & $(0) \rightarrow(+): 3$ cases & $0-5$ \\
\hline & & & $(+++): 1$ case & $30-50$ \\
\hline & Intralobular hyperplasia & 2 & $(++)$ & $30-60$ \\
\hline \multirow[t]{2}{*}{ Primary cancer in situ } & Ductal carcinoma & 5 & $(++)$ & $60-80$ \\
\hline & Lobular carcinoma & 2 & $(++) \rightarrow(+++)$ & $20-50$ \\
\hline \multirow[t]{3}{*}{ Invasive intraductal cancer } & Grade $\mathrm{I}^{\mathrm{b}}$ & 1 & $(+++)$ & 70 \\
\hline & Grade II $^{\mathrm{b}}$ & 4 & $(++) \rightarrow(+++)$ & $25-100$ \\
\hline & Grade III $^{\mathrm{b}}$ & 3 & $0 \rightarrow(+++)$ & $40-60$ \\
\hline Invasive lobular cancer & Lobular carcinoma & 2 & $(++)$ & $20-70$ \\
\hline Cancer metastasis & Metastasis cells of ductal carcinoma & 1 & $(++) \rightarrow(+++)$ & 100 \\
\hline \multirow{4}{*}{$\begin{array}{l}\text { Normal epithelial tissue } \\
\text { neighboring ductal and lobular } \\
\text { cancer lesions }\end{array}$} & In situ carcinoma & 2 & $(+++)$ & 40 \\
\hline & Invasive carcinoma & 8 & $(0): 4$ cases & 0 \\
\hline & & & $(+): 3$ cases & 5 \\
\hline & & & $(++): 1$ case & 40 \\
\hline
\end{tabular}

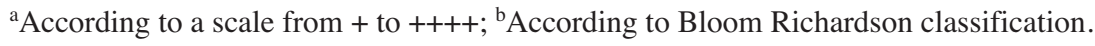

D-galactosyltransferase (22), but it is not known at which stage of this process functional changes of this enzyme occur.

In precedent study reports, we have demonstrated using direct fluorescent labeling that in pathologies considered as benign and in grade I of invasive ductal carcinomas, the binding site was essentially on the cell membrane, but that in grades II and III, the labeling involved the cytoplasm, and cell heterogeneity appeared.

In the present study, we used VVA- $\mathrm{B}_{4}$ lectin to investigate differences in Tn antigen expression in tissues of different types of human breast cancer (benign lesions, carcinoma in situ, invasive carcinoma) and in normal tissues neighboring ductal or lobular carcinoma, using an indirect HIC labeling to accurately determine the nature and localization of the labeling cells in these tissues.

\section{Materials and methods}

Breast tissues. Breast tissue specimens sampled during surgical procedures from 46 patients were provided by the Anatomical and Pathological Service of the Jean Perrin University Clinic in Clermont-Ferrand. The tissues showed benign hyperplastic changes (apocrine metaplasia, cylindrical metaplasia, and fibroadenoma), atypical hyperplasia, carcinoma in situ and invasive cancer (ductal and lobular forms). The invasive ductal tumors were graded according to the Bloom and Richardson classification (23). All cases were initially assessed by an expert pathomorphologist. Normal breast tissues collected from 3 non-pregnant woman aged between 17-29 years, originated from a private clinic performing mammoplastic procedures. The study material was divided into 7 categories: normal tissues, benign changes, atypical changes, primary cancer in situ, invasive ductal and lobular cancers, ductal cancer metastases to lymph nodes, and normal epithelial cells in the direct vicinity of lobular cancer (Table I).

The tissues were fixed with $4 \%$ formalin solution and embedded in paraffin. The $4 \mu \mathrm{m}$-thick sections were simultaneously analyzed by the conventional staining method (hematoxylin and eosin) and by an indirect immunohistochemical method utilizing dual antibodies and the streptavidinbiotin-peroxidase labeling system.

\section{Immunohistochemical method}

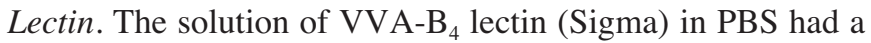
concentration of $2.5 \mu \mathrm{g} / \mathrm{ml}$. This lectin, isolated from Vicia villosa (Fabaceae) seeds, shows specificity for $\mathrm{N}$-acetyl- 

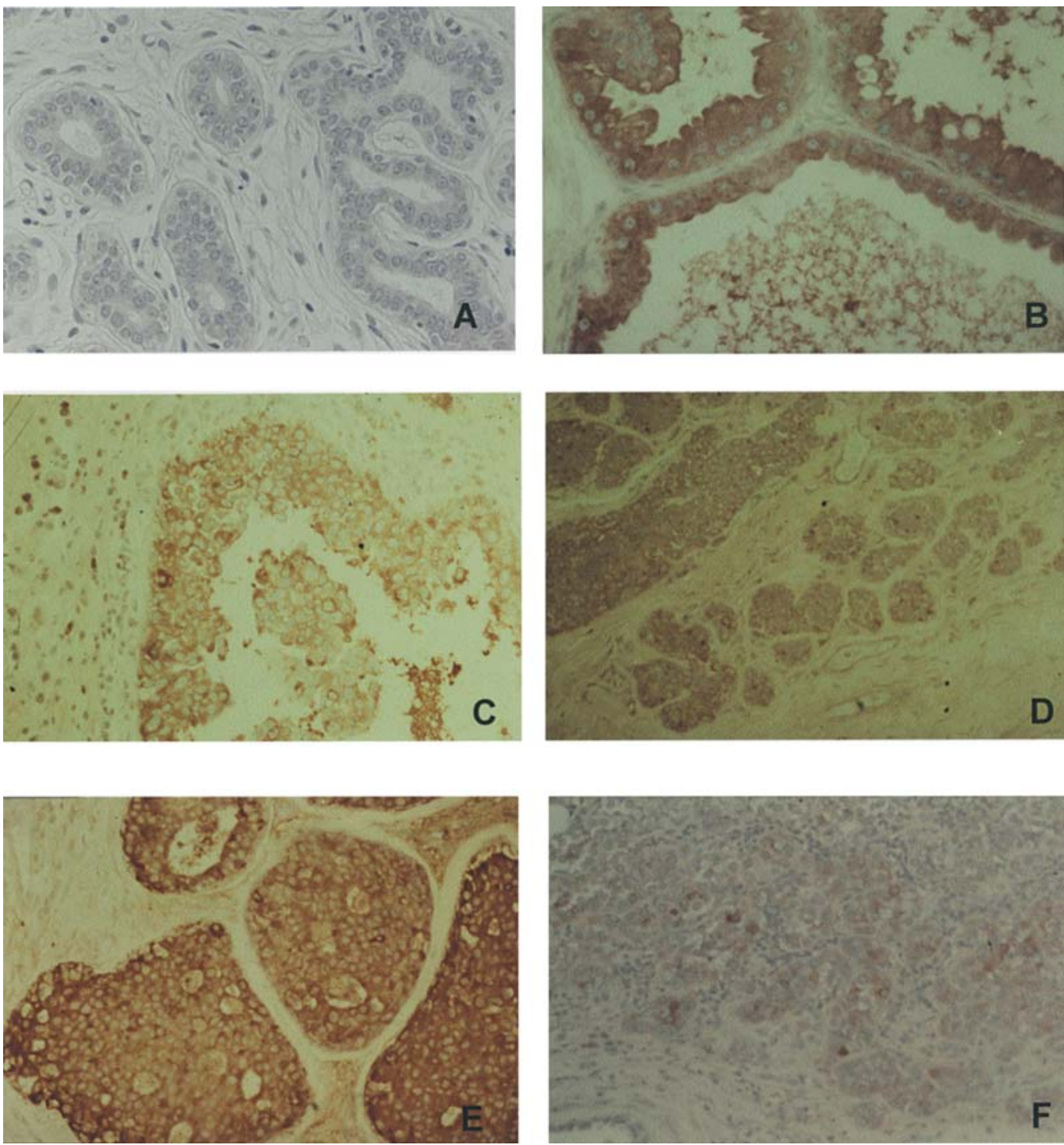

Figure 1. Tn antigen in benign changes and non-invasive primary cancer. A, Normal breast tissue from a mammoplastic procedure (x200). B, Apocrine metaplasia of three galactophores. Cells with strongly-stained cytoplasm on the apical pole (x320). C, Atypical ductal hyperplasia (x200). D, Atypical lobular hyperplasia (x200). E, Ductal in situ carcinoma. A majority of the cells were strongly stained (x200). F, Lobular in situ carcinoma. The heterogeneity of staining intensity (from + to +++ ) and of the number of labeled cells are clearly visible, with inflammatory infiltration negative (x200).

galactosamine (GalNAc) linked to the $\alpha$-O-glycoside bond with serine or threonine of the peptide chain, which corresponds to Tn antigen structure.

Antibodies. Rabbit anti-VVA-B $\mathrm{B}_{4}$ polyclonal antibodies were obtained by immunization of rabbits by systematic intravenous injections of the lectin bound with erythrocytes previously collected from the same rabbit. The appearance and specificity of the anti-lectin antibodies in the rabbit serum were monitored according to the Ouchterlony immunoprecipitation method.

Biotinylated goat anti-rabbit IgA monoclonal antibodies (Dako) were purchased as Universal LSAB 2 Kits together with the streptavidin-peroxidase system.

Streptavidin labeling system. Streptavidin-peroxidase complex and AEC (3-amino-9-ethylcarbazole), which is a peroxidase substrate, were purchased from Dako as ready-to-use reagent.

PBS-BSA buffer. Phosphate-buffered physiological saline containing $1 \%$ bovine serum albumin fraction V (MERCK) was used to saturate unspecific lectin and antibody binding sites.

Procedure. Following paraffin removal and hydration, the sections were incubated in $3 \%$ hydrogen peroxide for $10 \mathrm{~min}$ in order to deactivate endogenous peroxidase. They were subsequently washed once in distilled water, three times in PBS and twice in PBS-BSA, and incubated with lectin solution at room temperature for $1 \mathrm{~h}$. Then, the tissues washed in PBS-BSA were overlaid with rabbit serum containing antilectin antibodies (1-h incubation), biotinylated goat antibody (10-min incubation), streptavidin-peroxidase system (10 min) and 3-amino-9-ethylcarbasole (AEC), which is a peroxidase substrate $(10 \mathrm{~min})$. Before application of each reagent, the tissues were thoroughly rinsed with PBS-BSA. Finally, the sections were dipped in hematoxylin solution for $15 \mathrm{sec}$ and in water with several drops of ammonia to sharpen image contrast. Double negative control was processed simultaneously. Tissues taken as control were incubated with PBS-BSA buffer instead of lectin and rabbit antiserum. Inhibition tests were used to 
confirm specificity of VVA-B $\mathrm{B}_{4}$ binding to the sugar sequence corresponding to Tn antigen. This consisted in adding $0.1 \mathrm{M}$ $\mathrm{N}$-acetyl-D-galactosamine solution to the lectin solution before application to the tissues.

\section{Results}

The immunohistochemical method was able to localize sites of expression of Tn antigen sugar structure in the tissues, which were visualized as reddish-brown-stained cells. Staining intensity (expressed according to a four-grade scale), staining character (granular, dispersed), localization in the cell (cytoplasmatic or membrane labeling) were analyzed. In addition, an approximate percentage of labeled cells was calculated. The following observations were made in the cases divided into 7 basic categories (Table I).

1. Normal tissues. Among three cases under analysis, in two cases the reaction of epithelial cells was entirely negative. In the third case, the cytoplasm of about $15 \%$ of epithelial cells was very weakly stained (Fig. 1A).

2. Benign changes. Specimens with benign changes, caused by disturbances in the proliferation process, showed generally weak positive reactions, with differentiated staining intensity depending on the type of change: a) Cylindrical metaplasia: staining intensity was weak and number of stained cells did not exceed $20 \%$. b) Apocrine metaplasia: a large majority of cells (90\%) showed dispersed cytoplasmic staining, and staining intensity varied greatly between the cases (Fig. 1B). c) Fibroadenoma: nearly $4 \%$ of the cells showed moderate staining intensity.

3. Atypical changes. a) Atypical ductal hyperplasia: among four cases under investigation, three exhibited negative or almost negative reactions. In one case, cytoplasm staining of about $40 \%$ of the cells was intense (Fig. 1C). b) Atypical lobular hyperplasia: the cytoplasm of $30-60 \%$ of the cells was weakly stained (Fig. 1D).

4. Carcinoma in situ. a) Ductal carcinoma: the cytoplasm of more than half (60-80\% depending on the case) of the cells was moderately stained (++). Granular aggregations were sometimes clearly visible (Fig. 1E). b) Lobular carcinoma: $20-50 \%$ of the cells were strongly or moderately stained. Cytoplasm staining was noticeably heterogeneous (Fig. 1F).

5. Invasive carcinoma. a) Invasive ductal carcinoma: both number of stained cells and stain intensity varied depending on stage of tumor development (as per Bloom Richardson classification): Stage I, distinct strong staining of the cytoplasm of the majority of cancer cells (Fig. 2A). Stage II, number of stained cells and stain intensity differed strongly between cases. We observed that the cytoplasm of cells with a lower degree of transformation was more intensely stained (Fig. 2B). Stage III, at this stage of carcinogenesis, staining intensity became heterogeneous and there was a tendency towards lower numbers of stained cells (Fig. 2C). b) Invasive lobular carcinoma: from 20 to $70 \%$ of the cells were moderately stained (Fig. 2D).
6. Metastases of invasive ductal carcinoma. Specimens of axillary lymph nodes contained intensely-stained metastatic cells forming cords (Fig. 2E).

7. Normal epithelial cells located near in situ or invasive ductal and lobular cancer lesions. a) Primary carcinoma in situ: nearly half the normal epithelial cells located in close vicinity of cancer cells had intensely-stained cytoplasm (Fig. 2G and H). b) Invasive carcinoma: the reactions of the non-transformed cells varied among the 8 cases under study. In 4 cases, all normal cells presented negative reaction, in 3 cases only $5 \%$ of non-transformed cells showed weak reaction, and in 1 case, about $40 \%$ of the cells were moderately stained (Fig. 2F).

\section{Discussion}

Immunolabeling using VVA- $\mathrm{B}_{4}$ lectin and anti-lectin antibody allowed us to demonstrate the changes in the $O$-glycosylation process leading to the formation of GalNAc $\alpha-O$ serine/ threonine-type (Tn antigen) residues. Application of this method confirmed results obtained much earlier with procedures utilizing fluorochrome-bound lectin (FITC) $(19,20)$. Moreover, this method provides additional information about character and labeling localization (membrane, cytoplasmic, dispersed, condensed, granular), and allows both a preliminary quantification and establishment of the nature of the labeled cells.

Tissues collected during cosmetic procedures and classified as normal were completely negative in two cases, whereas a weak positive reaction of a very small number of cells seen in one case remains puzzling and difficult to explain. The labeling could have been caused by genetically-determined changes in glycosylation which did not carry any pathological consequences, although some early stage of cell pathology cannot be ruled out. Therefore, in view of proper and widespread prevention, it seems justified to also conduct systematic studies and identification of $\mathrm{T}$ and $\mathrm{Tn}$ antigen in tissues collected during cosmetic procedures.

Benign proliferative changes such as fibroadenoma and apocrine and cylindrical metaplasia usually showed a very weak positive reaction but there was a population of stronglylabeled cells. Numerous cells with strongly-stained cytoplasm were particularly conspicuous in apocrine metaplasia (Fig. 1B). The question as to whether the population of labeled cells will evolve towards neoplastic transformation could be definitively answered when this reaction is confirmed with other methods (e.g. using specific antibodies). Therefore, modifications of the glycoconjugate structures may precede the anomalies considered to be characteristic of malignant transformation, which is consistent with the existence of intermediate stages between normal and malignant states.

In non-invasive cases of atypical hyperplasia, labeling was distinct and depended on the site where this hyperplasia occurred. The ductal hyperplasia cells, except for one case (Fig. 1C), showed weaker labeling, while lobular hyperplasia was characterized by a relatively high percentage (up to 60\%) of cells showing moderate labeling (Fig. 1D).

A vast majority (up to $80 \%$ ) of in situ ductal and lobular carcinomas exhibited very strong or medium staining (Fig. 1E 

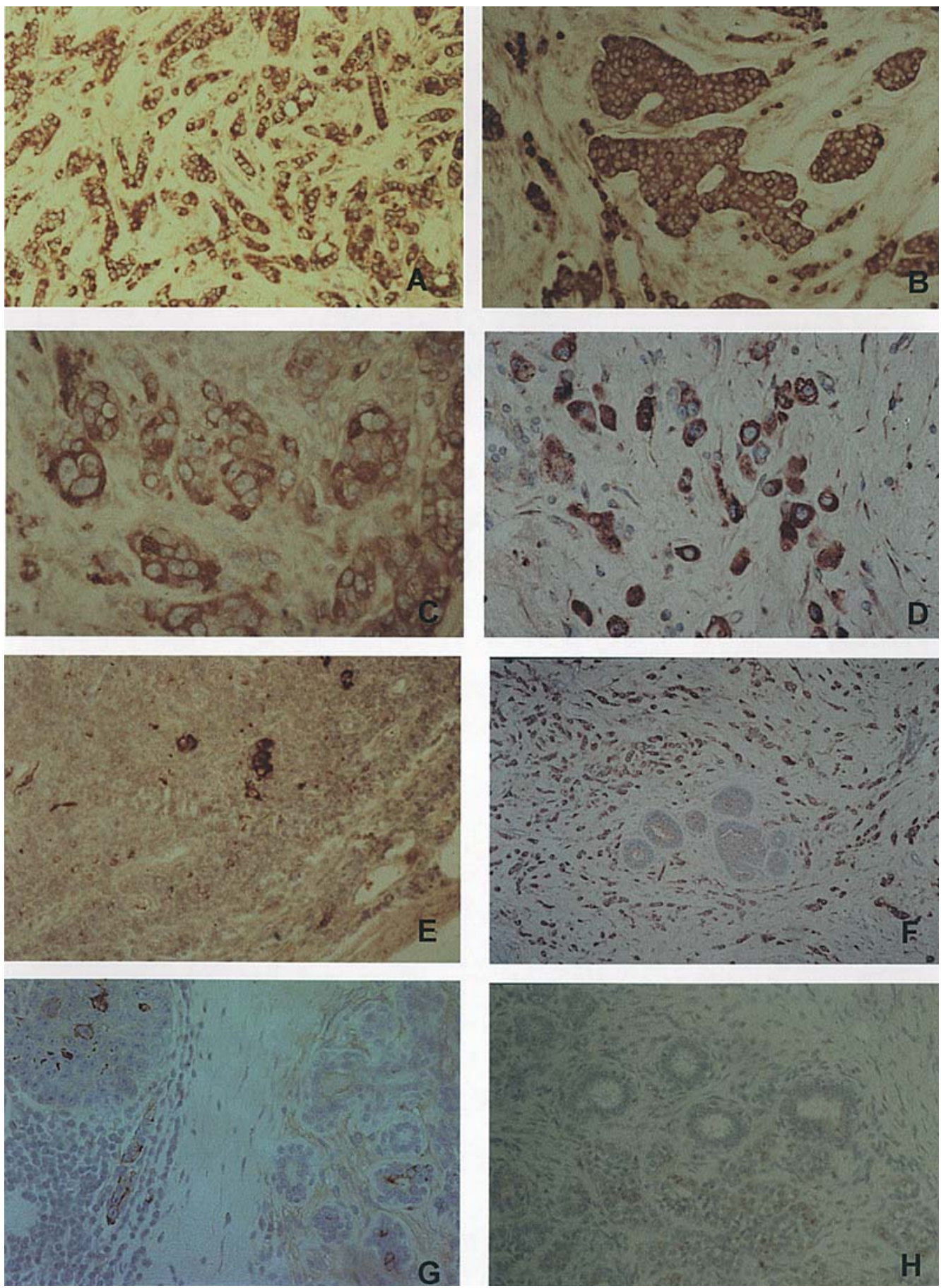

Figure 2. Tn antigen in invasive carcinoma and normal epithelial tissue neighboring ductal and lobular cancer. A, Invasive ductal cancer grade I. B, Invasive ductal cancer grade II (x200). Strong (+++) staining of almost all cells. C, Invasive ductal cancer grade III (x320). Besides strongly stained (+++) cells, negative (-) cancer cells are visible. D, Invasive lobular cancer (x450). Strong (+++), granular staining of the cytoplasm. E, Metastasis cells of ductal cancer to the axillary lymph node. Very strong cytoplasmic staining of the cancer cells (x225). F, Normal tissue in the vicinity of lobular invasive carcinoma (x200). Galactophore cells exhibit cytoplasm staining (++) in the apical region. G, Normal tissue in the vicinity of ductal in situ carcinoma (x200). H, Normal tissue in the vicinity of lobular in situ carcinoma (x200).

and F), with case-dependent labeling intensity, as was particularly noticeable in lobular carcinoma (Fig. 1F).

Labeling of the invasive cancers (ductal and lobular carcinoma) differed according to stage of cancer advancement, with a tendency towards lower numbers of cells in the most advanced stages. The heterogeneity in ductal carcinoma was seen beginning from stage II and progressing to a maximum at stage III, in which the number of labeled cells dropped to between 40 and $60 \%$ (Fig. 2B). The disappearance of all reactivity for a large proportion of cells at stage III may be due either to a loss of polypeptidyl-GalNAc transferase, which binds GalNAc to the protein backbone, or possibly to a lack of polypeptide synthesis, as has been suggested for colonic cancer in which the cells do not react with lectins or Tnspecific antibodies (9).

In the tissues located in the direct vicinity of in situ carcinoma, which did not show any morphological transformations, $40 \%$ of cells had labeled cytoplasm. This can be 
considered as a confirmation of a hypothesis suggesting the existence of 'an area of transformation' in which the cells receive a signal triggering neoplastic transformation which induces changes in the chemical structure of glycoconjugates. Further evidence for this hypothesis includes the observations reported by Itzkowitz et al (9) in colon cancer, demonstrating that $\mathrm{Tn}$, sialosyl-Tn and $\mathrm{T}$ antigens are expressed in the transitional mucosa without cellular atypia, immediately adjacent to the cancer. The findings with 'normal' tissue directly adjacent to invasive carcinoma were surprising, since these cells either did not show any positive reaction or the reaction was very weak (Fig. 2F). This can be explained by the rapid across-tissue penetration of cancer cells which had not yet transformed.

A single example of strongly reactive metastasis cells in intraductal cancer collected from the axillary lymph node is insufficient to draw any far-reaching conclusions. However, it should be emphasized that metastatic cells have similar phenotype as primary carcinoma cells.

In all the pathological cases, the labeling appeared to be membrane and cytoplasmic. This endoplasmic localization could be explained by alteration of abnormal glycoconjugate transport from the Golgi vesicles to the plasmalem, resulting in a cytoplasmic accumulation of the antigen. Moreover, the entire mucin-type glycoconjugate process is involved, and it is probable that the Tn antigen is likewise expressed by the internal membrane system.

It can be concluded that modifications in glycosylation manifested as the presence of Tn epitopes occur very early in neoplastic transformation, before destructive changes in proliferation/apoptosis or cell differentiation become observable. Tn antigen is therefore present in different forms of primary carcinoma and cancer metastases, while its structure disappears in very advanced stages.

The origin of Tn antigen and its influence on carcinogenesis have not yet been elucidated in detail. However, Tn antigen expression in breast carcinoma was proven not to be connected with a mutation leading to the blockade of T transferase, and it has been shown that different intermediate forms, e.g. antigen T, sTn and Tn, could be present on the same cancer cell (19).

\section{References}

1. Guillot J, Guerry M, Konska G, Caldefie-Chézet F, De Latour M and Penault-Llorca F: Modification des glycoconjugués au cours du processus de cancérisation: cas des carcinomes mammaires. Bull Cancer 91: 141-158, 2004.

2. Le Pendu J, Marionneau S, Cailleau-Thomas A, Rocher J, Le Moullac-Vaidye B and Clement M: ABH and Lewis histoblood group antigens in cancer. APMIS 109: 9-31, 2001.

3. Zuang D, Yousefi S and Dennis JW: Tn antigen and UDP-Gal: GalNAc $\alpha-R$ B1-3-galactosyltranferase expression in human breast carcinoma. Cancer Biochem Biophys 12: 185-198, 1991.

4. Julien S, Krzewinski-Recchi MA, Harduin-Lepers A, Gouyer V, Huet $G$ and Delannoy P: Expression of sialyl-Tn antigen in breast cancer cells transfected with the human CMP-Neu5Ac: GalNAc alpha2,6-sialyltransferase (ST6GalNAc I) cDNA. Glycoconj J 18: 883-893, 2001.
5. Barr N, Taylor CR, Young T and Springer GF: Are pancarcinoma $\mathrm{T}$ and Tn differentiation antigens? Cancer 64: 834-841, 1989.

6. Berger EG: Tn-syndrome. Biochim Biophys Acta 1455: 255-268, 1999.

7. Desai PR: Immunoreactive $\mathrm{T}$ and $\mathrm{Tn}$ antigens in malignancy: role in carcinoma diagnosis, prognosis, and immunotherapy. Transfus Med Rev 14: 312-325, 2000.

8. Zhang S, Zhang HS, Cordon-Cardo C, Reuter VE, Singhal AK, Lloyd KO and Livingston PO: Selection of tumor antigens as targets for immune attack using immunohistochemistry: II. Blood group-related antigens. Int J Cancer 73: 50-56, 1997.

9. Itzkowitz SH, Yuan M, Montgomery CK, Kjeldsen T, Takahashi HK, Bigbee WL and Kim YS: Expression of Tn, Sialosyl-Tn, and $\mathrm{T}$ antigens in human colon cancer. Cancer Res 49: 197-204, 1989.

10. Nakagoe T, Fukushima K, Tanaka K, Sawai T, Tsuji T, Jibiki M, Nanashima A, Yamagushi $\mathrm{H}$, Yasutake T, Ayabe $\mathrm{H}$ and Arisawa K: Evaluation of sialyl Lewis (a), sialyl Lewis (x), and sialyl Tn antigens expression levels as predictors of recurrence after curative surgery in node-negative colorectal cancer patients. J Exp Clin Cancer Res 21: 107-113, 2002.

11. Nanashima A, Yamagushi H, Nakagoe T, Matsuo S, Sumida Y, Tsuji T, Sawai T, Yamagushi E, Yasutake T and Ayabe H: High serum concentrations of sialyl Tn antigen in carcinomas of the biliary tract and pancreas. J Hepatobiliary Pancreat Surg 6: 391-395, 1999.

12. Nakagoe T, Sawai T, Tsuji T, Jibiki M, Nanashima A, Yamagushi $\mathrm{H}$, Yasutake $\mathrm{T}$ and Ayabe $\mathrm{H}$ : Pre-operative serum levels of sialyl Tn antigen predict liver metastasis and poor prognosis in patients with gastric cancer. Eur J Surg 27: 731-739, 2001.

13. Davidson B, Berner A, Nesland JM, Risberg B, Kristensen GB, Trope CG and Bryne M: Carbohydrate antigen expression in primary tumors, metastatic lesions, and serous effusions from patients diagnosed with epithelial ovarian carcinoma: evidence of up-regulated Tn and Sialyl Tn antigen expression in effusions. Hum Pathol 31: 1081-1087, 2000.

14. Carrilho C, Cantel M, Gouveia P and David L: Simple mucintype carbohydrate antigens (Tn, sialosyl-Tn, T and sialosyl-T) and gp230 mucin-like glycoprotein are candidate markers for neoplastic transformation of the human cervix. Virchows Arch 437: 173-179, 2000

15. Konska G, Guillot J, De Latour M and Fonck Y: Expression of Tn antigen and $N$-acetyllactosamine residues in malignant and benign human breast tumors detected by lectins and monoclonal antibody 83D4. Int J Oncol 12: 361-367, 1998.

16. Kishikawa T, Ghazizadeh M, Sasaki Y and Springer G: Specific role of $\mathrm{T}$ and $\mathrm{Tn}$ tumor-associated antigens in adhesion between a human breast carcinoma cell line and a normal human breast epithelial cell line. Jpn J Cancer Res 90: 326-332, 1999.

17. Tsuchiya A, Kanno M, Kawaguchi T, Endo Y, Zhang GJ, Ohtake T and Kimijima II: Prognostic relevance of Tn expression in breast cancer. Breast Cancer 25: 175-180, 1999.

18. Imai J, Ghazizadeh M, Naito Z and Asano G: Immunohistochemical expression of T, Tn and sialyl-Tn antigens and clinical outcome in human breast carcinoma. Anticancer Res 21: 1327-1334, 2001.

19. Konska G, Favy D, Guillot J, Bernard-Gallon D, De Latour M and Fonck Y: Expression des antigenes $\mathrm{T}$ et $\mathrm{Tn}$ dans les cancers du sein. C R Soc Biol 192: 733-747, 1998.

20. Konska G, Vissac C, Zagla K, Chézet F, Vasson M-P, BernardGallon D and Guillot J: Ultrastructural localization of binding sites for PNA and VVA-B4 lectins in human breast cancer cell lines detected by confocal fluorescence microscopy. Int J Oncol 21: 1009-1014, 2002.

21. Peumans WJ and van Damme EJM: Plant lectins: specific tools for the identification, isolation and characterization of $O$-linked glycans. Crit Rev Biochem Mol Biol 33: 209-258, 1998.

22. Thurnher M, Rusconi S and Berger EG: Persistent repression of a functional allele can be responsible for galactosyltransferase deficiency in Tn syndrome. J Clin Invest 91: 2103-2110, 1993.

23. Bloom HJ and Richardson WW: Histological grading and prognosis in breast cancer. Br J Cancer 11: 359-377, 1957. 